(1)

CrossMark

\title{
Adding an important piece to the pulmonary vascular resistance puzzle in pulmonary arterial hypertension
}

\author{
Bradley A. Maron ${ }^{1}$ and Marc Humbert $\mathbb{1}^{2}$ \\ Affiliations: 'Division of Cardiovascular Medicine, Brigham and Women's Hospital and Harvard Medical \\ School and Dept of Cardiology, Boston VA Healthcare System, Boston, MA, USA. ${ }^{2}$ Université Paris-Saclay, \\ INSERM UMR_S 999, AP-HP, Service de Pneumologie, Hôpital Bicêtre, Le Kremlin-Bicêtre, France.
}

Correspondence: Bradley A. Maron, 77 Ave Louis Pasteur, NRB Rm 0630-N, Boston, MA 02115, USA. E-mail: bmaronabwh.harvard.edu

@ERSpublications

Rethinking pulmonary vascular resistance in pulmonary arterial hypertension https://bit.ly/2RmnTDw

Cite this article as: Maron BA, Humbert M. Adding an important piece to the pulmonary vascular resistance puzzle in pulmonary arterial hypertension. Eur Respir J 2020; 56: 2000962 [https://doi.org/ 10.1183/13993003.00962-2020].

Clinical outcomes in pulmonary arterial hypertension (PAH) have improved substantially in the modern era, owing to greater clinician awareness, availability of numerous pulmonary vasodilator therapies and, now, more than two decades of sound clinical trial data informing optimal strategies for treating patients [1]. This arc of progress began with therapeutic interventions that aimed to simply delay mortality in patients with end-stage disease, at a time in which $\mathrm{PAH}$ was regarded as by-and-large uniformly fatal. The evolution of $\mathrm{PAH}$ into a contemporary and treatable disease has been marked by specific sentinel transition points, including proven efficacy of prescription exercise, sequential add-on treatment with different drug classes, and up-front combination therapy in newly diagnosed patients [2]. This has resulted in a collective shift toward greatly enhanced goals for defining treatment success in clinical practice, such as minimal symptom burden, preserved exercise tolerance, and favourable haemodynamic parameters [3].

In line with this trend, early PAH diagnosis has emerged as the next major front in the battle to optimise quality of life, decrease morbidity and improve upon hard clinical event rates that remain unacceptably elevated. This emphasis coincides with accumulating clinical and epidemiological research findings suggesting that opportunities may exist to refine the haemodynamic criteria defining pulmonary hypertension, generally, and $\mathrm{PAH}$, specifically $[4,5]$. Indeed, the upper limit of normal mean pulmonary artery pressure (mPAP) converges with data showing that decreased functional capacity and increased mortality in patients at risk for PAH begins at $\sim 20 \mathrm{mmHg}$ [6]. To modernise clinical practice, the 6th World Symposium on Pulmonary Hypertension (Nice, France) recommended changing the mPAP threshold defining PAH from $\geqslant 25 \mathrm{~mm}$ to $>20 \mathrm{mmHg}$ [7].

A principal objective of this revision was to capture patients with early-stage $\mathrm{PAH}$, for whom diagnosis and management is overlooked at present. Importantly, an increase in pulmonary vascular resistance (PVR) driven, at least in part, by plexigenic, fibrotic and hypertrophic effacement of pulmonary arterioles is a hallmark feature of PAH [2]. Yet, patients with mildly elevated mPAP are unlikely to meet the PVR threshold of $>3.0 \mathrm{WU}$ used to diagnose PAH currently, derived mainly from historical consensus opinion [8]. Thus, revising the mPAP threshold to $>20 \mathrm{mmHg}$ also established a need to clarify the optimal PVR for diagnosing PAH inclusive of early stage (mild) disease. Accomplishing this goal in PAH will need to 
draw on information framing PVR values that are normative, pathogenic and associated with favourable response to therapy.

In this issue of the European Respiratory Journal, RATwATTE et al. [9] add a key piece to the PVR puzzle by leveraging the unique clinical practice patterns in Australian and New Zealand, in which PVR $\geqslant 3.0 \mathrm{WU}$ is not obligatory for treating PAH. The investigators assembled data for $2378 \mathrm{PAH}$ patients enrolled in the PHSANZ registry between 2011 and 2018, and focused on 82 patients for whom the PVR was $<3.0 \mathrm{WU}$. This subgroup had the following haemodynamic profile (medians presented with interquartile ranges): PVR 2.2 (1.9-2.7) WU, mPAP 27 (IQR 25-30) mmHg, pulmonary artery wedge pressure 13 (11-14) $\mathrm{mmHg}$. All patients were prescribed at least an endothelin receptor antagonist (80.4\%) or phosphodiesterase type-V inhibitor (19.5\%), and $17 \%$ were on dual therapy. After a median follow-up of 5 months, 6-min walk distance increased on average by $46 \mathrm{~m}$ and one-third of patients improved $\geqslant 1.0$ New York Heart Association functional class, with greater effects observed in idiopathic rather than connective tissue disease-associated PAH.

Beyond these findings suggesting salutatory clinical benefit for the study population in the early phase of treatment, some important insights were gained during the long-term follow-up spanning a median 65 months after diagnosis. First, the PVR progressed to $>3.0 \mathrm{WU}$ in seven $(27 \%)$ of the 26 patients undergoing repeat right heart catheterisation at 1 year. This suggests that for some patients, PVR progresses to levels classically associated with $\mathrm{PAH}$ (i.e. $\geqslant 3.0 \mathrm{WU}$ ) soon after diagnosis, and this vulnerable subgroup may be captured earlier when considering a PVR range beginning $\sim 2.2 \mathrm{WU}$. Second, the 3-year and 5-year survival rates for the study population were $89 \%$ and $83 \%$, respectively, which are more favourable than for other real-world outcome data in PAH populations, including from studies with only dual therapy patients [10]. Taken together, these data begin an important narrative exploring potential opportunity to affect outcome in PAH when considering a PVR threshold $<3.0 \mathrm{WU}$.

As the authors assert, the study population was unique, but modest in size; when coupled with the retrospective study design, this may limit generalisability of the findings to other PAH populations. Further, results of this study do not clarify the optimal cardiopulmonary haemodynamic spectrum to define PAH, or that which should be used to initiate therapy. Indeed, patients with mPAP $21-24 \mathrm{mmHg}$, now included in the modern-day PAH definition, were not part of the current study. It is noteworthy, however, that PVR 2.0-2.2 WU has already been described as the upper limit of normal [5], and is associated with increased clinical events in large unselected populations [11] and connective tissue disease patients [12]. Nevertheless, data from this study are not ready for use in clinical practice, which as the authors clearly state will require further rigorous prospective investigations clarifying how best to interpret PVR $<3.0 \mathrm{WU}$ at point-of-care. Results extrapolated from this study could also be used to guide national screening programmes for detecting early stage $\mathrm{PAH}$, already underway in some countries, including France [13].

RATwATte et al. [9] are to be congratulated on these important data, which help build a much-needed framework for contemporising the PAH haemodynamic criteria relative to PVR. These findings should stimulate additional investigations using definitive clinical research methodologies, which were not yet available at the time the current work was assembled, to understand the association between PVR $<3.0 \mathrm{WU}$ and therapeutic response in PAH. It is in this way that the field moves toward further improving clinical outcome and, possibly, an era of preventative medicine.

Conflict of interest: B.A. Maron receives research funding from the NIH (NIH U01HL125215-01; R21HL134320; 1R01HL139613-01; U54HL119145), Cardiovascular Medical Research Education Foundation, and Boston Biomedical Innovation Center; is a co-inventor on US patent 9605047, US pending patent PCT/US2019/059890, and provisional patent applications 62475955 and 029672 ; and is a member of the steering committee for a research grant supported by Actelion Pharmaceuticals, outside the submitted work. M. Humbert reports grants and personal fees from Actelion, Bayer, GSK and Acceleron, personal fees from Merck and United Therapeutics, outside the submitted work.

Support statement: B.A. Maron receives research funding from the NIH (NIH U01HL125215-01; R21HL134320; 1R01HL139613-01; U54HL119145; R01HL153502), Cardiovascular Medical Research Education Foundation, and Boston Biomedical Innovation Center. Funding information for this article has been deposited with the Crossref Funder Registry.

\section{References}

1 Boucly A, Weatherald J, Savale L, et al. Risk assessment, prognosis and guideline implementation in pulmonary arterial hypertension. Eur Respir J 2017; 50: 1700889.

2 Maron BA, Galiè N. Diagnosis, treatment, and clinical management of pulmonary arterial hypertension in the contemporary era: a review. JAMA Cardiol 2016; 1: 1056-1065.

3 D'Armini AM, Ghofrani HA, Kim NH, et al. Use of responder threshold criteria to evaluate the response to treatment in the phase III CHEST-1 study. J Heart Lung Transplant 2015; 34: 348-355. 
4 Maron BA, Hess E, Maddox TM, et al. Association of borderline pulmonary hypertension with mortality and hospitalization in a large patient cohort: insights from the veterans affairs clinical assessment, reporting, and tracking program. Circulation 2016; 133: 1240-1248.

5 Kovacs G, Berghold A, Scheidl S, et al. Pulmonary arterial pressure during rest and exercise in healthy subjects: a systematic review. Eur Respir J 2009; 34: 888-894.

6 Condliffe R, Kovacs G. Identifying early pulmonary arterial hypertension in patients with systemic sclerosis. Eur Respir J 2018; 51: 1800495

7 Simonneau G, Montani D, Celermajer DS, et al. Haemodynamic definitions and updated clinical classification of pulmonary hypertension. Eur Respir J 2019; 53: 1801913.

8 Maron BA, Choudhary G, Tedford RJ, et al. Correspondence on the debate regarding the haemodynamic definition of pulmonary hypertension. Eur Respir J 2019; 53: 1900727.

9 Ratwatte S, Anderson J, Strange G, et al. Pulmonary arterial hypertension with below threshold pulmonary vascular resistance. Eur Respir J 2020; 56: 1901654.

10 Sitbon O, Sattler C, Bertoletti L, et al. Initial dual oral combination therapy in pulmonary arterial hypertension. Eur Respir J 2016; 47: 1727-1736.

11 Maron BA, Brittain EL, Hess E, et al. The association between pulmonary vascular resistance and clinical outcomes in patients with pulmonary hypertension: a retrospective cohort study. Lancet Respir Med 2020; in press.

12 Xanthouli P, Jordan S, Milde N, et al. Haemodynamic phenotypes and survival in patients with systemic sclerosis: the impact of the new definition of pulmonary arterial hypertension. Ann Rheum Dis 2020; 79: 370-378.

13 Montani D, Girerd B, Jais X, et al. Screening of pulmonary arterial hypertension in asymptomatic BMPR2 mutation carriers (DELPHI-2 Study). Eur Respir J 2019; 54: Suppl. 63, PA3951. 